\title{
Comparative analysis of pepper and tomato reveals euchromatin expansion of pepper genome caused by differential accumulation of Ty3/Gypsy-like elements
}

\author{
Minkyu Park ${ }^{1,2 \dagger}$, SungHwan Jo ${ }^{3,4 \dagger}$, Jin-Kyung Kwon ${ }^{2,5}$, Jongsun Park ${ }^{1,6,7,8}$, Jong Hwa Ahn 2,5, Seungill Kim,2, \\ Yong-Hwan Lee ${ }^{6,7,8}$, Tae-Jin Yang ${ }^{2,5}$, Cheol-Goo Hur ${ }^{4}$, Byoung-Cheorl Kang ${ }^{2,5}$, Byung-Dong Kim²,5 Doil Choi ${ }^{2,5^{*}}$
}

\begin{abstract}
Background: Among the Solanaceae plants, the pepper genome is three times larger than that of tomato. Although the gene repertoire and gene order of both species are well conserved, the cause of the genome-size difference is not known. To determine the causes for the expansion of pepper euchromatic regions, we compared the pepper genome to that of tomato.

Results: For sequence-level analysis, we generated $35.6 \mathrm{Mb}$ of pepper genomic sequences from euchromatin enriched 1,245 pepper BAC clones. The comparative analysis of orthologous gene-rich regions between both species revealed insertion of transposons exclusively in the pepper sequences, maintaining the gene order and content. The most common type of the transposon found was the LTR retrotransposon. Phylogenetic comparison of the LTR retrotransposons revealed that two groups of Ty3/Gypsy-like elements (Tat and Athila) were overly accumulated in the pepper genome. The FISH analysis of the pepper Tat elements showed a random distribution in heterochromatic and euchromatic regions, whereas the tomato Tat elements showed heterochromatinpreferential accumulation.

Conclusions: Compared to tomato pepper euchromatin doubled its size by differential accumulation of a specific group of Ty3/Gypsy-like elements. Our results could provide an insight on the mechanism of genome evolution in the Solanaceae family.
\end{abstract}

\section{Background}

The Solanaceae is an unusually divergent family consisting of approximately 90 genera and 3,000-4,000 species [1]. Members of the Solanaceae have evolved into extremely divergent forms, ranging from trees to annual herbs, and they occupy diverse habitats ranging from deserts to aquatic areas [1]. Such hyper-diversity in one family makes it useful to study plant adaptation and diversification. Despite this diversity, all Solanaceous species evolved during the last 40 million years [2].

\footnotetext{
* Correspondence: doil@snu.ac.kr

† Contributed equally

2Plant Genomics and Breeding Institute, Seoul National University, Seoul 151921, Korea

Full list of author information is available at the end of the article
}

Furthermore, almost all members share the same chromosome number $(\mathrm{x}=12)$ [2].

To date, diversity within the Solanaceae has been studied by comparative genome analyses using common genetic markers. As a result, we know that the Solanaceae genomes have undergone relatively small numbers of chromosomal rearrangements (e.g., about 5 rearrangements between potato and tomato and about 30 rearrangements between pepper and tomato), maintaining well-conserved gene content and order [3-8]. The conservation of the Solanaceae genic region was also identified by the comparison of a syntenic segment in eggplant, pepper, petunia and tomato [7].

Despite such conservation, the genome sizes of the Solanaceae family members are diverse. For example, the genome size of the Solanum tuberosum (potato) is

C Biomed Central

(c) 2011 Park et al; licensee BioMed Central Ltd. This is an Open Access article distributed under the terms of the Creative Commons Attribution License (http://creativecommons.org/licenses/by/2.0), which permits unrestricted use, distribution, and reproduction in any medium, provided the original work is properly cited. 
$840 \mathrm{Mb}$, S. lycopersicum (tomato) $950 \mathrm{Mb}$, Petunia hybrida (petunia) $1200 \mathrm{Mb}$, and Capsicum annuum (pepper) $2700 \mathrm{Mb}$. However, the genetic analyses conducted to date were not successful at explaining genome size diversity due to limitations in the genetic markers. Hence, a sequence-level analysis to investigate the cause of the genome size diversity is required.

Among the Solanaceous species, pepper and tomato show strong advantages for the study of genome size difference because of following reasons. First, the genome size of pepper is three times larger than that of tomato. Second, the duplication of the whole genome did not occur during the evolution of both species [8]. Third, although pepper and tomato show large size differences in their genomes, their speciation is estimated to have occurred recently (approximately 16.2-22.2 million years ago) [7], which makes them not as closely related as potato and tomato, but more closely related than tobacco and tomato within the Solanaceae family [9]. Therefore, the investigation of genome diversity between pepper and tomato can represent the general trend of genome diversification among Solanaceous members that have not undergone the whole genome duplication.

To date, most studies related to the pepper genome have been carried out by generating genetic maps $[6,10-14]$. In contrast, the structure of the tomato euchromatic and heterochromatic regions has been the subject of several studies through the analyses of tomato BAC sequences [15-17]. Furthermore, the tomato genome sequencing project is currently underway, with the goal of generating a reference genome in the Solanaceae [18-21].

As a first study concerning the expansion of the pepper genome, the present work addresses the causes behind the expansion of pepper euchromatic regions. For this purpose, $35.6 \mathrm{Mb}$ of pepper sequences from 1,245 BAC clones selected from euchromatin-enriched regions were generated. Using information from the tomato genome project, $39.9 \mathrm{Mb} \mathrm{BAC}$ sequences of tomato were chosen for comparing orthologous generich sequences and the constitution of repetitive elements between the pepper and tomato genomes. We used fluorescence in situ hybridization (FISH) to support the results. This study presents an example of the Solanaceae genome diversity revealing how the pepper euchromatic region was expanded.

\section{Results}

\section{Sequencing of pepper BAC clones}

To produce the pepper sequence data representative of all pepper euchromatic regions, 1,235 pepper BAC clones of an average insert size of $130 \mathrm{~Kb}$ [22] were sequenced using pyrosequencing technology. To enrich the euchromatic regions, BAC clones were selected by BAC screening using labelled cDNAs derived from pepper mRNAs (extracted from flower, fruit, stem and leaf). A total of $90.8 \mathrm{Mb}$ of assembled sequences was obtained from $18.22 \times$ coverage sequences generated by 454 GS FLX-Titanium (454 Life Science, Roche). To avoid the bias caused by the short contig length, we used the long contigs, whose length is over $30 \mathrm{~Kb}$ (total length is 34.6 $\mathrm{Mb}$ ), in the analyses (Figure 1). In addition, ten selected pepper BAC clones containing gene-rich regions of pepper chromosome 2 were sequenced using Sanger methods, resulting in a total of 985,237 bp contig sequences (see Additional file 1). Three of the ten BAC sequences were assembled into one contig, resulting in a total of eight full-contig BAC sequences. These eight full-contig $B A C$ sequences were used in the comparative microsynteny analysis of pepper and tomato euchromatic regions.

\section{Comparison of visible genome structures}

Prior to the comparative sequence analysis between pepper and tomato, we analyzed visible chromosome structures in pepper and tomato using pachytene chromosomes. On visual inspection, the pepper and tomato chromosomes showed differences in structure. The tomato heterochromatic regions were mainly located on the pericentromeric regions and the euchromatic regions were clearly distinct from the heterochromatin structure (Figure 2A). In contrast, the pepper pachytene chromosomes showed more extensive

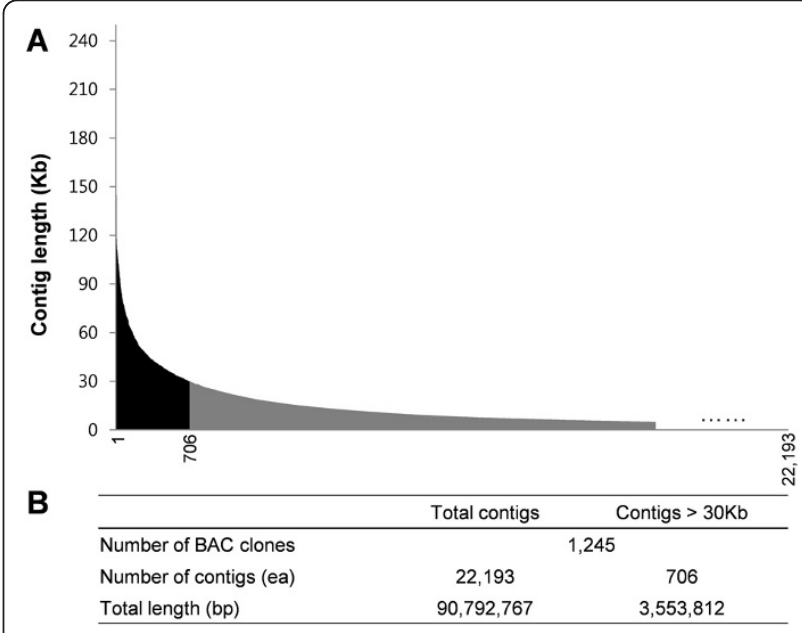

Figure 1 Information about 1,245 pepper BAC sequences. (a) Histogram of the assembled contig sizes. The contigs longer than $30 \mathrm{~Kb}$ are depicted by a black area and the shorter contigs are shown in gray. The contigs longer than $5 \mathrm{~Kb}$ are depicted in this histogram. (b) Information about contig number and total length. A total of 706 out of 22,193 contigs were longer than $30 \mathrm{~Kb}$ and their total length was about $35.6 \mathrm{Mb}$. This $35.6 \mathrm{Mb}$ sequence was used in the analysis. 

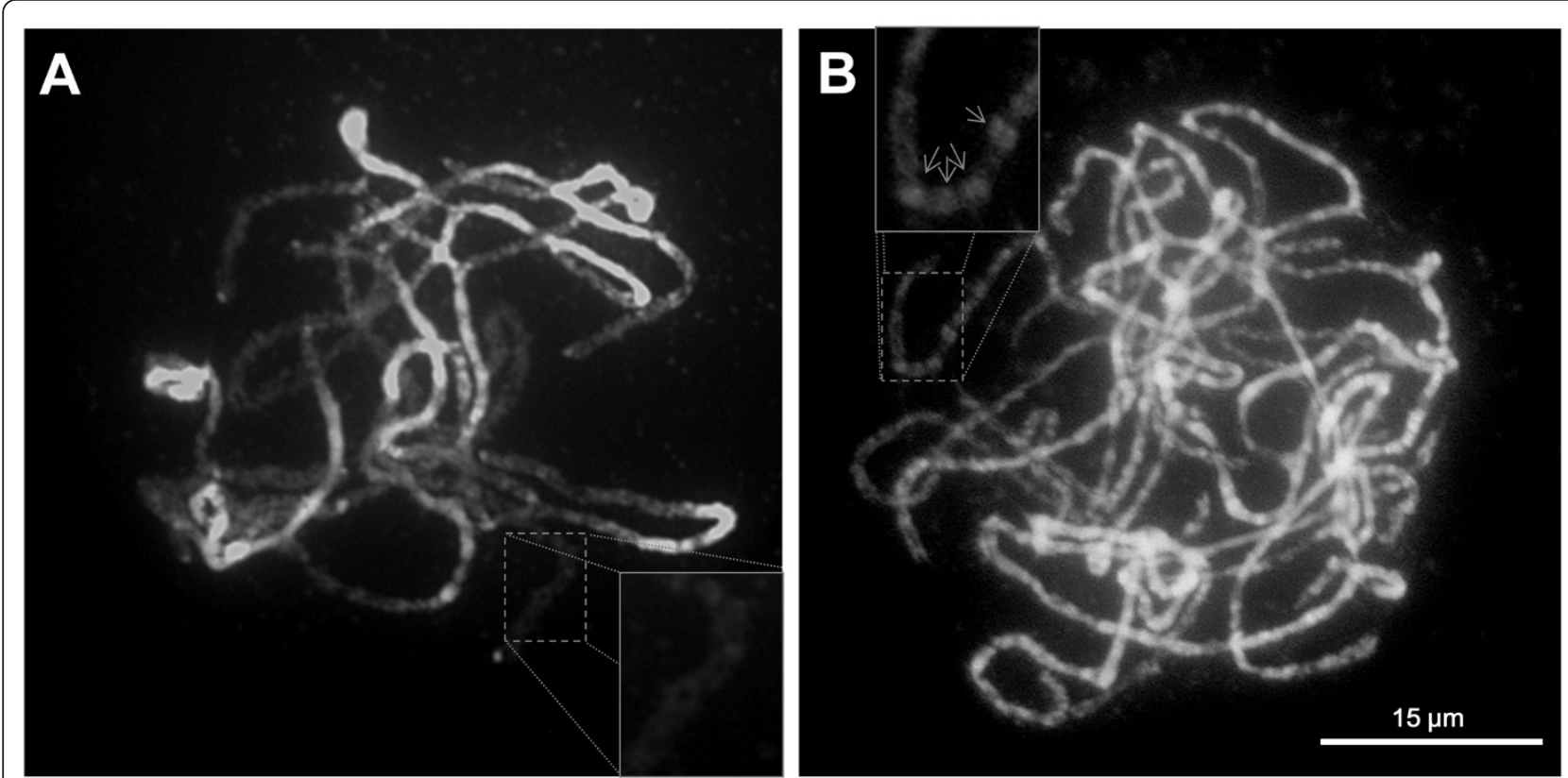

Figure 2 Microscopic structures of pachytene chromosomes of tomato (a) and pepper. (b). The pachytene chromosomes were stained with DAPI and the images were converted to black and white. The heterochromatic and euchromatic regions are shown as bright and dark lines, respectively.

heterochromatic regions (Figure 2B). Furthermore, the pepper euchromatic regions were intermixed with the heterochromatin structure (Figure 2B; indicated by arrows).

\section{Comparison of repetitive elements in the orthologous gene-rich regions}

To investigate the reasons for the presence of differences in euchromatin structure between both genomes, the orthologous gene-rich sequences of pepper and tomato were compared. To compare within the same chromosome, the orthologous gene-rich sequences were selected in chromosome 2 that has no inter-chromosomal crossover between both species [8]. BAC sequences distributed over seven positions in chromosome 2 were used to avoid bias based on position within the chromosome (Figure 3 and Additional file 1). The positions of the BAC sequences were determined using genetic markers on the tomato genetic map (tomato-EXPEN 2000, http://sgn.cornell.edu/) [23]. On the basis of tomato chromosome 2 , the centromere is located at the top $(0$ $\mathrm{CM}$ ) of the tomato genetic map (Figure 3) [15]. Eight orthologous pepper BAC sequences of a total of 985,237 bp were compared with the tomato sequences consisting of 490,745 bp (Table 1).

The comparative analysis of the orthologous gene-rich sequences revealed many insertions found exclusively in the pepper sequences. The insertions were transposable elements, and there were 35 transposable elements in the compared pepper sequences (Figure 3; colored boxes and Additional file 2). Boundary of the LTR-retrotransposons was determined in 16 elements by manual inspection (Figure 3; marked with asterisks in the colored boxes). The else transposons were found by gene prediction and repeat BLAST search in Repbase $[24,25]$. All of the transposable elements were found in the inter-genic regions, therefore without a disruption of the other structural genes. The insertion of the transposable elements resulted in a doubling of the pepper sequence size in comparison to that of tomato. Accordingly, the gene density was lower in pepper $(13,136 \mathrm{bp}$ per one gene) than in tomato $(7,011 \mathrm{bp}$ per one gene).

To determine the most prevalent type of transposon, the composition of the repetitive elements found in the compared sequences was analyzed. By repeat BLAST search in Repbase, a total of 191,393 bp of transposon sequences were found in pepper and 44,336 bp in tomato. The repeat sequences were classified into three groups (Figure 4).

Among identified transposable elements, LTR-retrotransposon sequences were the most abundant. Most of the LTR-retrotransposons were found in the pepper sequences (Figure 4). In addition, 28 of the 35 transposable elements found in pepper sequences were identified as LTR retrotransposons. The pepper sequences contained LTR-retrotransposon sequences with a frequency approximately 22 times higher than in the tomato sequences. The other two repeat classes also 


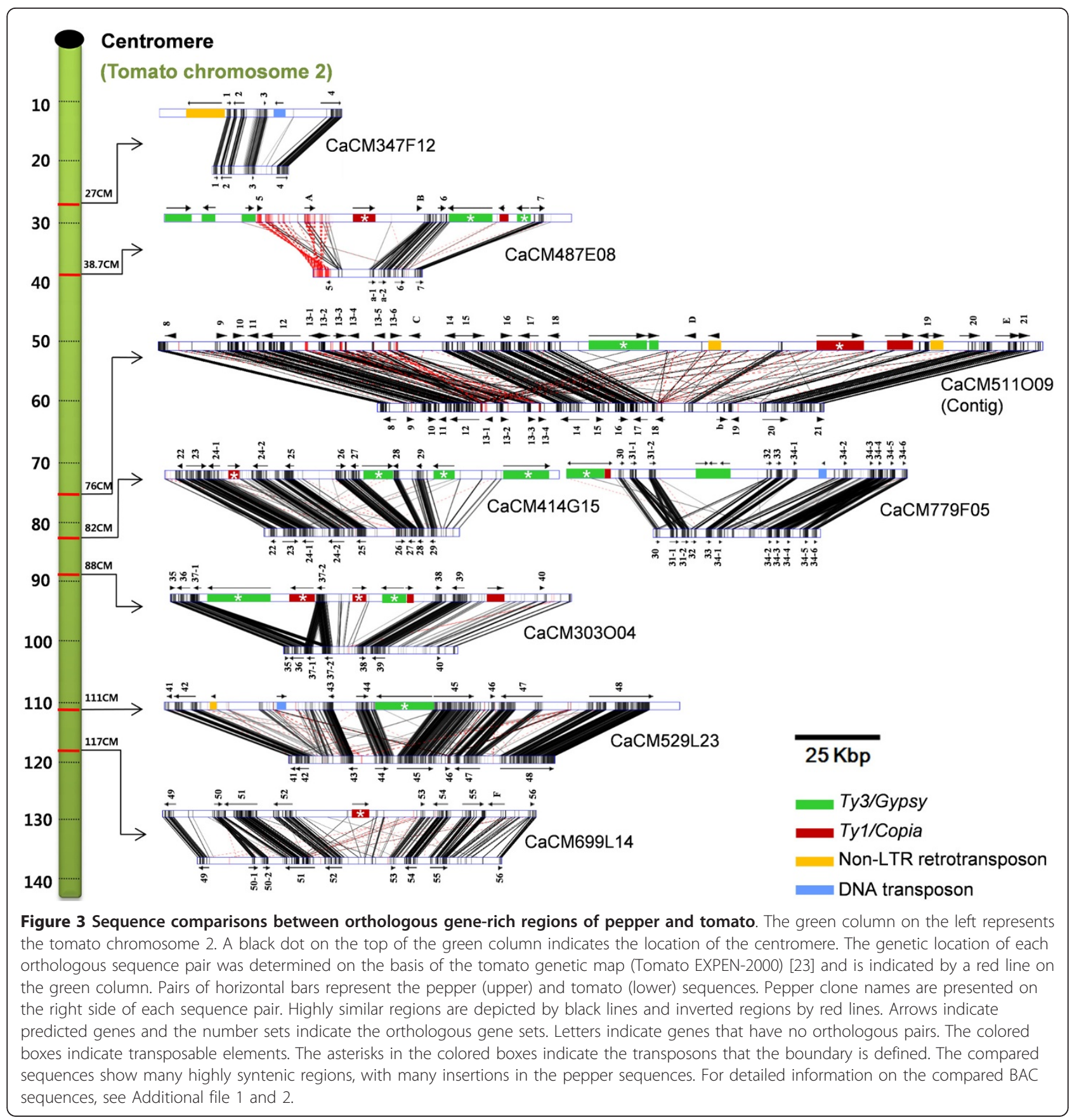

presented higher proportions in pepper than in tomato. Pepper had about 1.7 times as many DNA transposons, 4 times the number of non-LTR retrotransposons (Figure 4).

According to our transposon annotation results, the total length of the transposons found was 210,341 bp (see Additional file 2). Among them, Ty3/Gypsy-like element was the most abundant as 134,523 bp in total length (approximately 64\% of the annotated repeats), which suggests its important role in pepper euchromatin expansion. The next was Ty1/Copia-like elements as 55,173 bp (approximately $26 \%$ of the annotated repeats). The Non-LTR retrotransposon and DNA transposon was $12,159 \mathrm{bp}$ and $5,486 \mathrm{bp}$, respectively.

\section{Similar gene composition between pepper and tomato}

In contrast to the repetitive elements, gene constitution less affected the difference in the sequence size. In the compared sequences, a total of 145 genes were predicted excluding the transposable element genes. These 
Table 1 Statistics of the compared pepper and tomato gene-rich sequences

\begin{tabular}{|c|c|c|c|}
\hline & Pepper & Tomato & $\begin{array}{l}\text { Total } \\
\text { (ratio) }\end{array}$ \\
\hline $\begin{array}{l}\text { Total length of compared } \\
\text { sequence }\end{array}$ & $985,237 \mathrm{bp}$ & $490,745 \mathrm{bp}$ & - \\
\hline Number of predicted genes & 75 & 70 & 145 \\
\hline $\begin{array}{l}\text { Total length of predicted } \\
\text { genes }\end{array}$ & $247,338 \mathrm{bp}$ & $195,342 \mathrm{bp}$ & - \\
\hline Gene density & $\begin{array}{l}13,136 \mathrm{bp} / \\
\text { gene }\end{array}$ & $\begin{array}{l}\text { 7,011 bp/ } \\
\text { gene }\end{array}$ & - \\
\hline $\begin{array}{l}\text { Genes paired into orthologous } \\
\text { set }\end{array}$ & 69 & 67 & $136(94 \%)$ \\
\hline Genes that have no ortholog & 6 & 3 & $9(6 \%)$ \\
\hline Duplicated genes & 18 & 19 & $37(25 \%)$ \\
\hline $\begin{array}{l}\text { Average length of coding } \\
\text { region }\end{array}$ & $1,366 \mathrm{bp}$ & 1,332 bp & - \\
\hline Average length of intron & $1,815 \mathrm{bp}$ & $1,459 \mathrm{bp}$ & - \\
\hline
\end{tabular}

included 75 pepper genes and 70 tomato genes (Table 1 ). The total length of the genes combined was $247,338 \mathrm{bp}$ in pepper and 195,342 bp in tomato, showing a length difference of $51,996 \mathrm{bp}$. The total gene-length difference corresponded to approximately $10 \%$ of the total length difference of the compared sequences. The total genelength difference was mainly caused by the intronlength difference. A total of 136 out of 145 genes were paired into 56 orthologous sets (see Additional file 2). In these sets the average length of the pepper coding regions was $1,366 \mathrm{bp}$, which was $34 \mathrm{bp}$ longer than that of tomato $(1,332 \mathrm{bp})$, whereas the average intron length in pepper was $1,815 \mathrm{bp}$, which was $356 \mathrm{bp}$ longer than that of tomato $(1,459 \mathrm{bp})$. Among the 56 orthologous sets, six sets $(10.7 \%)$ consisted of duplicated genes. These six sets corresponded to 37 of the 145 genes (25\%), of which 18 genes were in pepper and 19 in

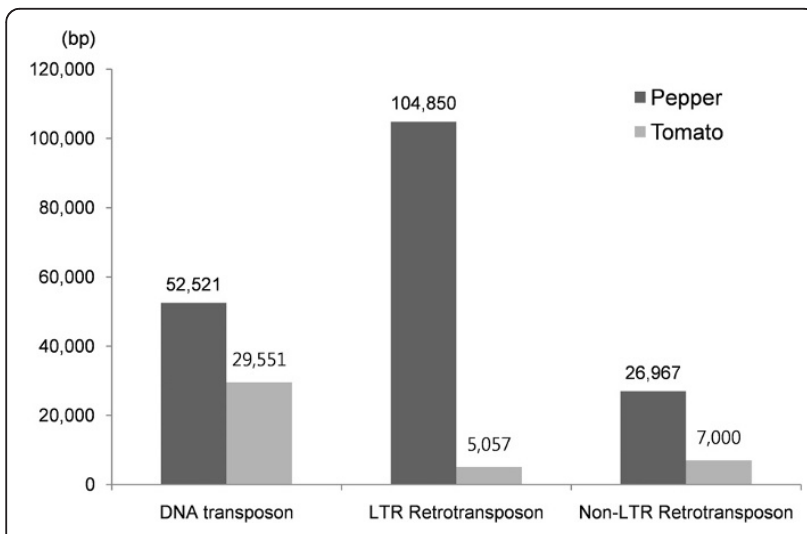

Figure 4 Analysis of the compared clones for repetitive elements. Three kinds of repetitive elements in the pepper and tomato BAC clones were compared by the total length. Among the repetitive elements, the pepper LTR retrotransposon shows the most significant difference. tomato (see Additional file 3). Hence, there was no remarkable bias in gene duplication number between both species (Table 1).

\section{Identification of LTR retrotransposons in the pepper and tomato genome sequences}

The causes for the accumulation of LTR retrotransposons in the pepper euchromatic regions were investigated by comparing the overall constitution of LTR retrotransposons between pepper and tomato by phylogenetic analysis. For this analysis, reverse transcriptase (RT) sequences, which are constitutive genes in LTR retrotransposons [26], were identified from the pepper and tomato genome sequences. The RTs were classified into Ty3/Gypsy and Ty1/Copia types by BLAST search in Repbase [24,25]. A total of 155 Ty3/Gypsy-like and 166 Ty1/Copia-like tomato RTs were identified from $39.9 \mathrm{Mb}$ of tomato BAC sequences (http://sgn.cornell. edu/about/tomato_sequencing.pl; downloaded in August, 2008) and 312 Ty3/Gypsy-like and 48 Ty1/Copia-like pepper RTs were found in the $35.6 \mathrm{Mb}$ pepper BAC sequences. Because the tomato genome project focused on the gene-rich region, the number of heterochromatin-preferential LTR retrotransposons might be underestimated in this comparison.

\section{Differential accumulation of a group of Ty3/Gypsy-like elements}

The phylogenetic tree of Ty3/Gypsy-like elements was generated by 312 pepper and 155 tomato RTs. A total of three subgroups were clearly identified from the phylogenetic tree (Figure 5). Each subgroup was classified on the basis of reported elements by BLAST search against GyDB (http://gydb.org/). The BLAST results with high confidence (e-value below e-40) were used for classification reference [27]. The representative elements of each subgroup which are acquired from the GyDB were also included in the phylogenetic tree. According to the classification, the three groups belonged to Tat and Athila subgroups, which belong to Athila/Tat, and to Del subgroup, which belongs to chromoviruses. Most of the Ty3/Gypsy-like elements were found in the three major subgroups.

The Ty3/Gypsy-like elements in the Del subgroup were identified as being accumulated in the pericentromeric heterochromatin. Yang et al. reported that the PCRT1 in the Del subgroup is a tomato Ty3/Gypsy-like element distributed throughout pericentromeric heterochromatin of tomato [16]. This result was consistent with our FISH result of another tomato Del element (Figure 5; indicated by yellow triangle). Furthermore, the FISH result of the pepper Del element exhibited the same distribution pattern as that of tomato (Figure 5; indicated by purple triangle). These results suggest that 


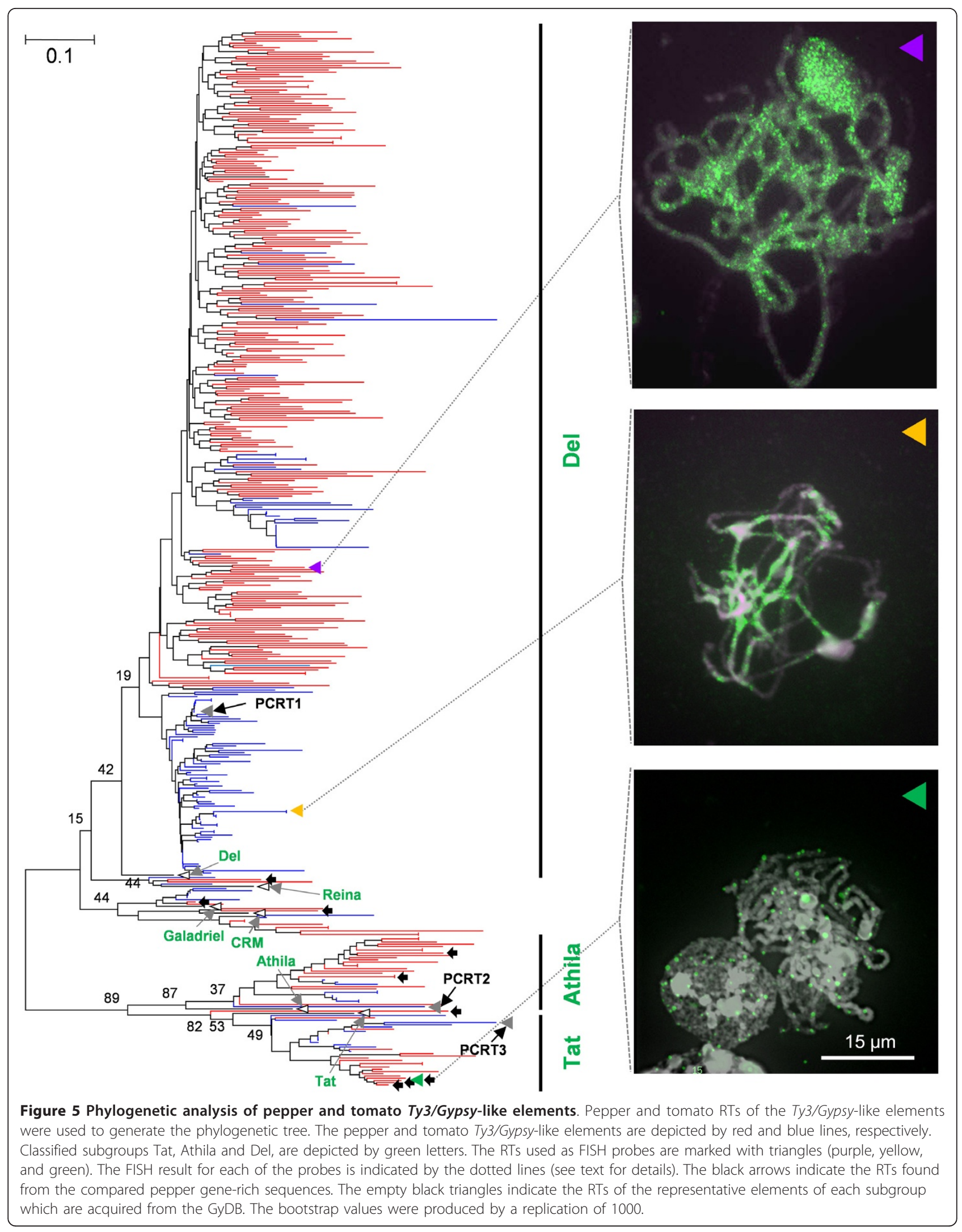


the Del elements constitute pericentromeric heterochromatin in both genomes, which means they do not affect euchromatin expansion.

The differences that may affect the expansion of pepper euchromatic regions were observed in the Tat and Athila subgroups. The number of pepper Tat and Athila elements was approximately twice the number in tomato (42 in pepper and 23 in tomato). According to the previous report by Yang et al., PCRT2 and PCRT3 are the tomato Ty3/Gypsy-like elements preferentially distributed in the tomato heterochromatic regions [16]. These two elements belonged to Athila and Tat respectively, suggesting that the tomato Ty3/Gypsy-like elements in these groups are accumulated in heterochromatic regions. In contrast, the FISH result of the pepper Tat element showed randomly distributed signals throughout the pepper chromosomes including the euchromatic regions (Figure 5; indicated by green triangle). Furthermore, four of the nine black arrows indicating the pepper Ty3/Gypsy-like elements found in the compared pepper gene-rich sequences belonged to Tat. Likewise, two of the nine elements belonged to the Athila subgroup, indicating the elements in this group are also found in pepper gene-rich regions. However, the Del subgroup didn't contain any of the Ty3/Gypsy-like elements found in the pepper gene-rich sequences (Figure 5). These results show that, in contrast to the distribution in tomato, the pepper Ty3/Gypsy-like elements in the Tat and Athila subgroups are randomly inserted throughout the whole genome, including the euchromatic regions.

\section{Chromodomains in the Ty3/Gypsy-like elements}

A chromodomain functions to recognize the heterochromatic regions when the Ty3/Gypsy-like elements insert into chromosomes $[28,29]$. To determine the chromatin selectivity of the Ty3/Gypsy-like elements, the existence of the chromodomain was investigated in each group. For this analysis, 72 intact Ty3/Gypsy-like elements were identified from the pepper and tomato sequences to check the chromodomain (see Additional file 4). Except for the Tat and Athila elements, almost all of the other intact Ty3/Gypsy-like elements contained the chromodomain (Figure 6A; filled dots, Additional file 5). The existence of the chromodomain in the Del intact elements was consistent with the heterochromatin-preferential accumulation of the Del elements in both species. Likewise, the absence of the chromodomain in Tat and Athila was consistent with the random accumulation of pepper elements. However, the absence of the chromodomain was in disagreement with the anticipated heterochromatin-preferential accumulation of tomato Tat and Athila elements.

To determine whether the tomato Tat and Athila elements are really accumulated in the heterochromatic regions in sequence-level, we investigated gene densities of the 17 tomato BAC sequences that contain the Tat and Athila elements (see Additional file 6). Two of the 17 tomato BAC sequences were gene-rich regions with a gene density similar to that of the compared tomato gene-rich sequences (Figure 7). However, the remaining 15 BAC sequences were gene-poor regions, in which the minimum gene density was about three times lower than that of the compared tomato gene-rich sequences. Considering that the tomato sequences are mainly from euchromatic regions, the accumulation of the tomato Tat and Athila elements shows a bias toward the heterochromatic regions. This result was consistent with the heterochromatin preferential distributions of the PCRT2 and PCRT3, indicating that the tomato Tat and Athila elements are accumulated in heterochromatic regions without the chromodomain.

\section{Proportion of the intact pepper Ty3/Gypsy-like elements in the genome}

The proportion of the pepper Ty3/Gypsy-like elements in the genome was estimated using the intact LTR retrotransposons (see methods for detail) (Figure 6B). The proportion of the individual elements was broadly different according to the classified groups. The average proportion of the Tat elements was $1.28 \%$ but the Athila was $0.64 \%$, suggesting more active accumulation of the Tat elements. The elements in the Del showed higher proportion than other classified groups in the genome as $2.01 \%$ of average proportion.

\section{Highly diversified features with similar lineage collections} of Ty1/Copia-like elements

The phylogenetic tree of Ty1/Copia-like elements presented highly diversified features that differed from those of Ty3/Gypsy-like elements. However they also showed similar lineage collections between pepper and tomato. By blast search against GyDB, four subgroups of the Ty3/Copia-like elements, Tork, Sire, Oryco, and Retrofit, were classified (Figure 8). The Tork was constituted with four subgroups that match to Fourf, Tork4, Tnt-1, and Batata. The six pepper Ty1/Copialike elements found in the eight orthologous pepper BAC sequences are indicated by black arrows in Figure 8. These six elements belonged to diverse phylogenetic positions in the phylogenetic tree (Figure 8). One of the six pepper elements that belongs to the Retrofit was tested by FISH analysis, and the signals distributed randomly on the chromosomes (Figure 8; indicated by red triangle). The FISH signals of the tomato Ty1/ Copia-like element that belongs to the Tork4 of the Tork subgroup were also observed in both brightly and darkly stained chromosome regions, indicating its distribution in heterochromatic and euchromatic regions 


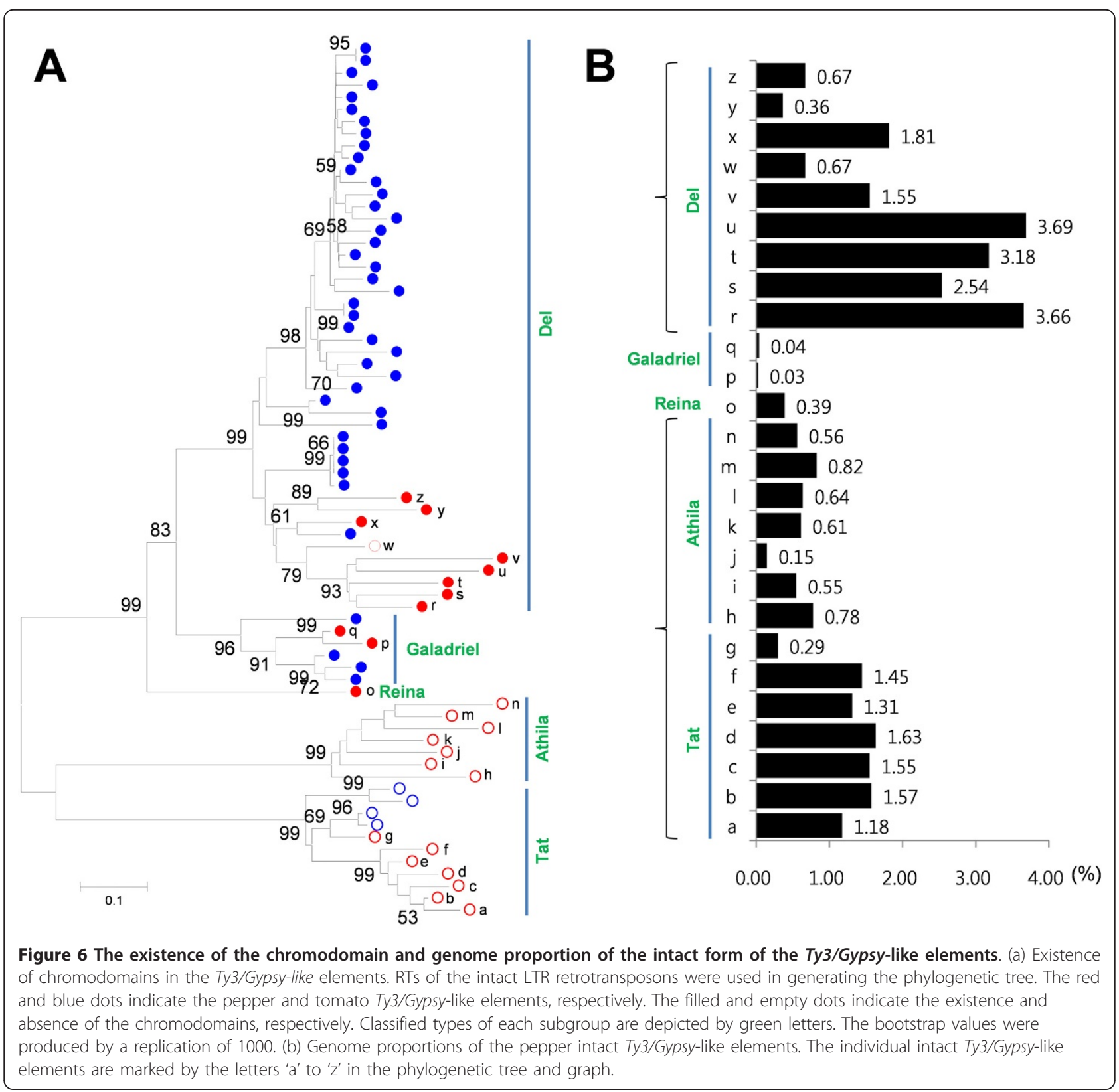

(Figure 8 ; indicated by blue triangle). On the other hand, the FISH signals of tomato Batata element in the Tork subgroup and the elements in the Sire subgroup were observed mainly in the brightly stained chromosome regions, indicating a heterochromatin-preferential distribution (Figure 8; indicated by orange and pink triangle).

\section{Discussion}

The results of the present study revealed that one of the important factors for the expansion of pepper euchromatic regions was the massive accumulation of the pepper Tat and Athila elements. In the Tat and Athila subgroups, the Ty3/Gypsy-like elements were found to be approximately two times more abundant in pepper than tomato. Considering that the pepper sequences used in this study were smaller than those of tomato in terms of total length (three-quarters of tomato) and in each contig length (Figure 1), the number of pepper Tat and Athila elements would further exceed that of tomato. Given that the tomato Tat and Athila elements preferentially accumulated in the heterochromatic regions (Figure 7), the higher copy number and random insertion of the pepper Tat and Athila elements suggests their important role in the expansion of pepper euchromatic regions. 


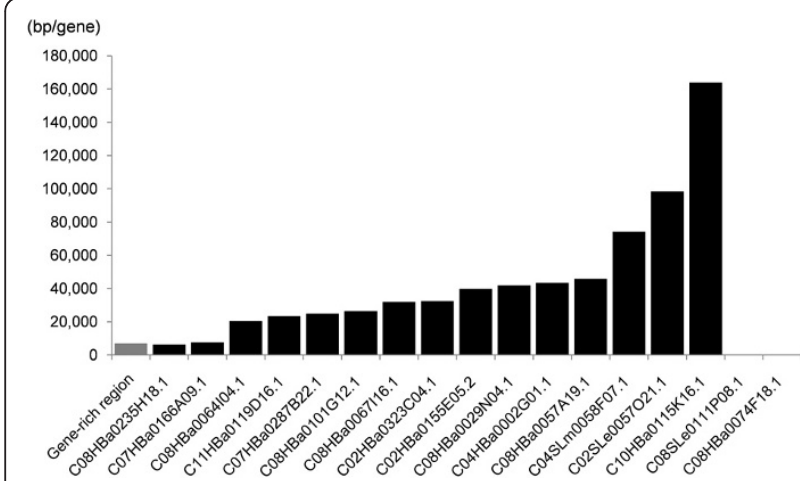

Figure 7 The gene density of the tomato BAC sequences containing the Tat and Athila elements. Gene-density of the seventeen tomato BAC sequences containing the Tat and Athila elements is presented. The gene density of the 'Gene-rich region' depicted by the gray column indicates the average gene density of the compared tomato gene-rich sequences. The gene number was counted with the exception of the transposable element genes. The gene density of the fifteen BAC sequences was lower than that of the gene-rich region by at least three times. The gene-density of the remaining two BAC sequences was similar to that of the generich region. No genes were found in the C08SLe0111P08.1 and C08HBa0074F18.1.

According to the FISH analyses, the Del elements in both pepper and tomato genomes were identified as forming the pericentromeric heterochromatin blocks. Unlike the Ty1/Copia-like elements, the Ty3/Gypsy-like elements that constitute pericentromeric heterochromatin blocks are known to be selectively inserted into the heterochromatic regions in A. thaliana [30]. The existence of the chromodomain in both pepper and tomato Del intact elements can explain this insertion selectivity. The insertion site preferences of LTR retrotransposons have also been observed in other plant genomes, including conifers, and members of the genus Helianthus $[31,32]$. Although the number of Del elements is predominant in the phylogenetic tree, accumulation of the Del elements would have expanded the pericentromeric heterochromatin, not affecting euchromatin expansion in both species.

Pereira reported that the Ty1/Copia-like elements in A. thaliana were randomly inserted into the whole genome, after which they underwent purifying selection in euchromatic regions [30]. This resulted in the preferential accumulation of the Ty1/Copia-like elements in the pericentromeric heterochromatin blocks of $A$. thaliana genome. In the present study, a similar phenomenon was detected in the heterochromatin preferential accumulation of the tomato Ty3/Gypsy-like elements that belong to Tat and Athila (Figure 7). The absence of the chromodomain in the elements indicates its initial random insertions in the genome. However, the purifying selection may have been eliminated the tomato Tat and
Athila elements from the tomato euchromatic regions, resulting in their preferential accumulation in the heterochromatic regions.

The comparative analysis of the LTR retrotransposons in the present study revealed a similar collection of lineages in both Ty3/Gypsy-like and Ty1/Copia-like elements. Given that the LTR retrotransposons of the same lineage have similar characteristics, both genomes would have accumulated the Ty1/Copia-like elements and Ty3/ Gypsy-like elements of the Tat and Athila in their euchromatic regions. However, the copy number of the elements in the tomato euchromatic regions may have been reduced due to the purifying selection, which could have resulted in the lower accumulation of the LTR retrotransposons in the tomato euchromatic regions than in those of pepper.

The number of pepper Del elements corresponded to twice the number of tomato Del elements (256 in pepper and 122 in tomato). This difference was partially due to the euchromatin selective sequencing of the tomato genome project. Although the pepper BAC clones were selected by the labelled cDNAs of mRNAs, our bioinformatics survey suggested that a large portion of pepper BAC clones contained heterochromatic regions. This phenomenon can be caused by contamination with the transcripts of repetitive elements, such as retrotransposons, during the selection of the BAC clones.

The expansion of genome sizes through the accumulation of LTR retrotransposons is well documented among flowering plants [30,33-35]. Based on the results of the present study, the expansion of the pepper genome is also due to the accumulation of LTR retrotransposons. A similar comparative analysis of repetitive elements in close species was carried out between $A$. thaliana and Brassica oleracea by Zang et al. [36]. Zang et al. reported that the large size of the $B$. oleracea genome is accounted by the higher copy number of each type of transposable elements within a similar collection of lineages, explaining the overall genome expansion [36]. However, the gene densities of both genomes were 4.5 $\mathrm{kb} /$ gene in A. thaliana and $6.6 \mathrm{~kb} /$ gene in B. oleracea [37], indicating that the euchromatic regions of both genomes are highly gene-rich, as is the case in tomato. In contrast with $B$. oleracea, pepper has an expanded euchromatin structure, and the present results explained the expansion of the euchromatic regions. Hence, the comparison of the pepper and tomato genomes can provide new insights into the expansion of euchromatic regions by the accumulation of repetitive elements.

\section{Conclusions}

The results of the present study show that the Ty3/ Gypsy-like elements in the Tat and Athila play an 


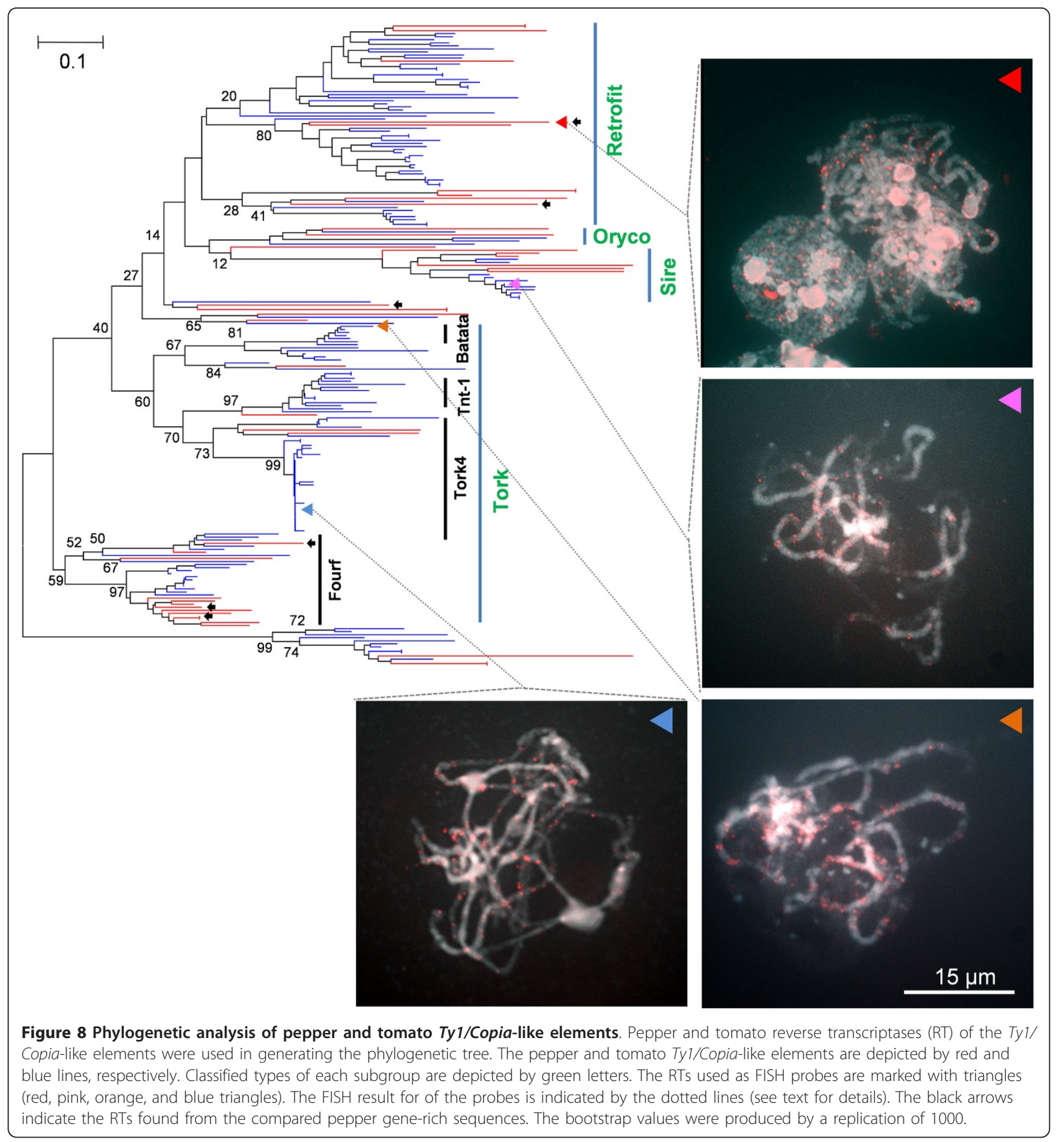

important role in the expansion of the pepper euchromatic regions. The genic regions of pepper and tomato were found to be well conserved with regards to gene order and content. However, the euchromatic regions in pepper were expanded to twice the size of those in tomato, mainly due to the insertion of LTR retrotransposons. The LTR retrotransposons in the pepper euchromatic regions may also explain why the pepper euchromatic regions look like intermixed with the heterochromatin structures.

\section{Methods}

Sequencing of the pepper and tomato BAC clones

The selection of pepper BAC clones for the comparison of the orthologous gene-rich regions was performed as described in this section: seven tomato BAC sequences 
that are distributed on chromosome 2 were chosen. The tomato sequences were used as queries in a BLASTN search of the pepper EST database to find orthologous pepper ESTs. Two or three pepper ESTs that were orthologous to sequences about $30 \mathrm{~kb}$ apart in each tomato BAC clone were used as probes. The probes were labelled via PCR amplification using specific primers and ${ }^{32}$ P-labeled dCTP. Seven or eight labelled probes were pooled, and the Southern hybridizations were carried out on pepper BAC library filters. The filters were sequentially washed in $2 \mathrm{X}$ SSC for $60 \mathrm{~min}, 1$ X SSC for $60 \mathrm{~min}$, and $0.5 \mathrm{X}$ SSC $60 \mathrm{~min}$. The positive clones were confirmed by colony PCR using the same primers. The pepper BAC clones that showed positive PCR results were used for sequencing. Each BAC clone was fully sequenced and analyzed by NICEM (http:// nicem.snu.ac.kr/) using the ABI 3730xl system (Applied Biosystems Inc $[\mathrm{ABI}]$, Foster City, CA). From each pepper BAC clone, a shotgun sequencing library was constructed using the pUC118 vector with an average insert size of 3-5 kb. BigDye Terminator chemistry version 3.1 (ABI) was used for the sequencing reactions. All of the sequences were analyzed by Phred/Pharp/Consed processing [38]. Base-calling and assembling of the sequences were carried out using the Phred/Phrap software. The Phred scores of the sequences were 30 or higher. The assembled sequences were edited using Consed software. Sequence editing for consensus contig formation was carried out using the Sequencher 4.1.5 (Gene codes Corp., Ann Arbor, USA). The tomato BAC sequences were generated by the same method as part of the International Tomato Sequencing Project of Korea [20].

A total of 1,235 pepper BAC clones were additionally selected for next-generation sequencing using 454 GS FLX-Titanium (454 Life Science, Roche). Each BAC clone DNA was manually extracted and normalized. The normalized BAC clone DNAs were pooled into 125 clones per a reaction channel of 454 GS FLX-Ti. Each sequencing reaction of 454 GS FLX-Ti was divided into two. The sequencing procedures for the 454 GS FLX-Ti were carried out using manufacturer-supplied protocols and reagents. The sequences were assembled by Newbler 2.0.1. The average coverage of the contigs was $18.22 \times$. Among the assembled contigs, the contigs longer than $30 \mathrm{~kb}$ were used in the analyses.

\section{Gene prediction and comparative analysis}

For accurate gene structure analysis, we predicted genes using three steps as follows: (1) Genes were predicted by FGENESH using a trained data set from tomato [39]. (2) The predicted genes were confirmed by BLASTP searches of the GenBank database (http://www.ncbi.nlm. nih.gov/) using the protein sequences of the predicted genes as queries. Among the predicted genes, those with scores greater than 100 and e-values less than e-20 were used in the next step. (3) Among the BLASTP results for each predicted gene, the protein sequence that had the highest score was chosen as a reference, and the gene models were predicted again by FGENESH+ using the trained data set from tomato. These results were used as gene models for the pepper and tomato sequences. The visualization of compared orthologous sequences was carried out using GATA [40] with minimum bits of 30 and maximum bits of 35.6. The repeat sequences were found by BLAST search in the Repbase repeat masking (http://girinst.org/censor/index.php) $[24,25]$.

\section{Phylogenetic analysis of LTR retrotransposons}

The RTs were found by Hmmer 2.1 [41] using the RTs reported in the Pfam database (http://pfam.sanger.ac.uk/, Accession No. PF00078 and PF07727) as a training set. The super family of the RTs was determined by the Rebase repeat masking. The RTs were confirmed by BLASTP searches in the GenBank database, and the RTs with a score over 200 were used in the analyses. The RTs containing any frame shift mutations or deletions were manually deleted in the alignment. Intact LTR retrotransposons were predicted using LTR_FINDER [42] with the default settings or by manual inspection using DOT-PLOT analysis. These analyses were conducted in the environment of the Comparative Fungal Genomics Platform (CFGP; http://cfgp.snu.ac.kr/) [43].

The phylogenetic trees were generated using the MEGA 4.0 software [44]. The alignments were carried out using ClustalW of MEGA 4.0 with the default settings (see Additional file 7, 8 and 9). The aligned RT sequences were used for generating the phylogenetic trees. The Poisson correction model and Neighbor-Joining method were used, and the phylogeny test was carried out by bootstrapping with 1,000 replications.

\section{Chromodomain search and proportion calculation of the intact LTR retrotransposon in the genome}

The chromodomains were found by BLASTX search of the intact LTR retrotransposons with the chromodomain proteins in the Pfam database (PF00385). The chromodomains found in the intact LTR retrotransposons were used again in finding the chromodomains in the other sequences. The intact LTR retrotransposons that have no chromodomain were confirmed again by the conserved domain search service in the NCBI (http://www.ncbi.nlm.nih.gov/Structure/cdd/wrpsb.cgi).

The proportions of the individual intact LTR retrotransposons in the pepper genome were calculated using the 90.8 Mbp of the assembled sequence and 3.2 Mbp 
of additional BAC sequences of pepper. By BLASTN search against the total $94 \mathrm{Mbp}$ pepper sequence, total matched sequence length of each intact LTR retrotransposon was calculated. The threshold e-value of the search was e-5. The total matched sequence length of the individual intact LTR retrotransposon was divided by the $94 \mathrm{Mbp}$ of pepper sequence size.

\section{FISH analysis}

The FISH probes for analyzing the LTR retrotransposons were produced by PCR amplification using the primer sets listed in Additional file 10 online. Pachytene chromosomes of tomato (Lycopersicon esculentum cv. Micro-Tom) were prepared according to the methods of Koo et al. [45]. Metaphase and pachytene chromosomes of pepper (Capsicum annuum cv. CM334) were prepared as described by Kwon et al. [46]. All probes were labelled with biotin 16-dUTP or digoxygenin 11-dUTP by nick translation as described by the manufacturer's protocol (Roche, Germany). The FISH experiments for tomato and pepper were performed according to the methods described by Koo et al. [45] and Kwon et al. [46], respectively. The hybridization solutions contained $50 \%$ formamide $(\mathrm{w} / \mathrm{v}), 10 \%$ dextran sulfate $(\mathrm{w} / \mathrm{v}), 5 \mathrm{ng} / \mu \mathrm{l}$ salmon sperm DNA, and $20 \mathrm{ng}$ of each probe in $2 \mathrm{X}$ SSC. The probes were detected using fluorescein avidin DCS (Roche, Germany) and rhodamine anti-digoxygenin (Roche, Germany). Pachytene chromosomes were counterstained with DAPI $(1 \mathrm{mg} / \mathrm{ml})$ in Vectashield antifade (Vector Laboratories). All images were captured and analyzed using the DeltaVision imaging system and associated software (Applied Precision, USA) with a cool SNAP CCD camera at NICEM. All images were improved for optimal brightness and contrast using Adobe Photoshop.

\section{Additional material}

Additional file 1: The compared BAC sequence sizes and GenBank
accession numbers.
Additional file 2: Information about predicted genes of the
compared sequences.
Additional file 3: Information about duplicated genes.
Additional file 4: Sequence information of the 72 intact Ty3/Gypsy-
like elements.
Additional file 5: List of chromodomains isolated from intact
pepper and tomato LTR retrotransposons.
Additional file 6: Information about predicted genes in the 17
tomato BAC sequences containing Tat and Athila elements.
Additional file 7: Alignment of reverse transcriptases of Ty3/Gypsy-
like elements.
Additional file 8: Alignment of reverse transcriptases isolated from
intact pepper and tomato LTR retrotransposons.
Additional file 9: Alignment of reverse transcriptases of Ty1/Copia-
like elements.

Additional file 10: List of PCR primers used to produce the FISH probes.

\section{Abbreviations}

BAC: bacterial artificial chromosome, EST: expressed sequence tag, FISH: fluorescence in situ hybridization, LTR: long terminal repeat, NGS: nextgeneration sequencing, RT: reverse transcriptase

\section{Acknowledgements}

This research was supported by a grant from Crop Functional Genomics Center (CG1132) of the 21st Century Frontier Research Program and National Research Foundation (Project No. 2010-0015105) funded by the Ministry of Education, Science and Technology of Republic of Korea.

\section{Author details}

${ }^{1}$ Interdisciplinary Program in Agriculture Biotechnology, Seoul National University, Seoul 151-921, Korea. ${ }^{2}$ Plant Genomics and Breeding Institute, Seoul National University, Seoul 151-921, Korea. ${ }^{3}$ Seeders Inc., Daejeon, Korea. ${ }^{4}$ Bioinformatics Research Center, KRIBB, Daejeon, 305-806, Korea. ${ }^{5}$ Department of Plant Science, Seoul National University, Seoul, 151-921, Korea. ${ }^{6}$ Fungal Bioinformatics Laboratory, Seoul National University, Seoul 151-921, Korea. ${ }^{7}$ Center for Fungal Pathogenesis, Seoul National University, Seoul 151-921, Korea. ${ }^{8}$ Department of Agricultural Biotechnology, Seoul National University, Seoul 151-921, Korea.

\section{Authors' contributions}

MP designed this study, carried out the overall sequence analysis, and wrote the draft manuscript. SJ generated the tomato sequence data and helped the manuscript revise. JKK carried out the fluorescence in situ hybridization. JP assembled the pepper sequence data and helped sequence analysis. JHA carried out pepper BAC clone sequencing. SK participated in the pepper sequence assembly. JP, YHL, TJY, CGH, BCK, and BDK helped the manuscript revise. DC conceived the study, helped to draft the manuscript, and organized the sequencing of pepper and tomato BAC sequences. All authors read and approved the final manuscript.

Received: 27 February 2010 Accepted: 29 January 2011

Published: 29 January 2011

\section{References}

1. Knapp S, Bohs L, Nee M, Spooner DM: Solanaceae - a model for linking genomics with biodiversity. Comp Funct Genomics 2004, 5:285-291.

2. Wikström N, Savolainen V, Chase MW: Evolution of the angiosperms: calibrating the family tree. Proc Biol Sci 2001, 268:2211-2220.

3. Bonierbale MW, Plaisted RL, Tanksley SD: RFLP Maps Based on a Common Set of Clones Reveal Modes of Chromosomal Evolution in Potato and Tomato. Genetics 1988, 120:1095-1103.

4. Tanksley SD, Bernatzky R, Lapitan NL, Prince JP: Conservation of Gene Repertoire but not Gene Order in Pepper and Tomato. Proc Natl Acad Sci 1988, 85:6419-6423.

5. Prince JP, Pochard E, Tanksley SD: Construction of a molecular linkage map of pepper and a comparison of synteny with tomato. Genome 1993, 36:404-417.

6. Livingstone KD, Lackney VK, Blauth JR, van Wijk R, Jahn MK: Genome mapping in Capsicum and the evolution of genome structure in the Solanaceae. Genetics 1999, 152:1183-1202.

7. Wang Y, Diehl A, Wu F, Vrebalov J, Giovannoni J, Siepel A, Tanksley SD: Sequencing and comparative analysis of a conserved syntenic segment in the Solanaceae. Genetics 2008, 180:391-408.

8. Wu F, Eannetta NT, Xu Y, Durrett R, Mazourek M, Jahn MM, Tanksley SD: A COSIl genetic map of the pepper genome provides a detailed picture of synteny with tomato and new insights into recent chromosome evolution in the genus Capsicum. Theor Appl Genet 2009, 118:1279-1293.

9. Bohs L, Olmstead RG: Phylogeneti relationships in Solanum (Solanaceae) based on ndhF sequences. Syst Bot 1997, 22:5-17.

10. Kang BC, Nahm SH, Huh JH, Yoo HS, Yu JW, Lee MH, Kim BD: An interspecific (Capsicum annuum $\times C$. chinese) F2 linkage map in pepper using RFLP and AFLP markers. Theor Appl Genet 2001, 102:531-539. 
11. Lefebvre V, Pflieger S, Thabuis A, Caranta C, Blattes A, Chauvet J, Daubèze A, Palloix A: Towards the saturation of the pepper linkage map by alignment of three intraspecific maps including known-function genes. Genome 2002, 45:839-854.

12. Lee JM, Nahm SH, Kim YM, Kim BD: Characterization and molecular genetic mapping of microsatellite loci in pepper. Theor Appl Genet 2004 108:619-627.

13. Paran I, van der Voort JR, Lefebvre V, Jahn M, Landry L, van Schriek $M$, Tanyolac B, Caranta C, Chaim AB, Livingstone K, Palloix A, Peleman J: An integrated genetic linkage map of pepper (Capsicum spp.). Mol Breeding 2004, 13:251-261.

14. Minamiyama $Y$, Tsuro N, Hirai M: An SSR-based linkage map of Capsicum annuum. Mol Breeding 2006, 18:157-169.

15. Koo DH, Jo SH, Bang JW, Park HM, Lee S, Choi D: Integration of Cytogenetic and Genetic Linkage Maps Unveils the Physical Architecture of Tomato Chromosome 2. Genetics 2008, 179:1211-1220.

16. Yang TJ, Lee $S$, Chang SB, Yu Y, de Jong H, Wing RA: In-depth sequence analysis of the tomato chromosome 12 centromeric region: identification of a large CAA block and characterization of pericentromere retrotranposons. Chromosoma 2005, 114:103-117.

17. Wang Y, Tang X, Cheng Z, Mueller L, Giovannoni J, Tanksley SD: Euchromatin and pericentromeric heterochromatin: comparative composition in the tomato genome. Genetics 2006, 172:2529-2540.

18. Mueller LA, Solow TH, Taylor N, Skwarecki B, Buels R, Binns J, Lin C, Wright MH, Ahrens R, Wang Y, Herbst EV, Keyder ER, Menda N, Zamir D, Tanksley SD: The SOL Genomics Network: a comparative resource for Solanaceae biology and beyond. Plant Physiol 2005, 138:1310-1317.

19. Asamizu E: Tomato genome sequencing: deciphering the euchromatin region of the chromosome 8. Plant Biotechnol J 2007, 24:5-9.

20. Lee S, Jo SH, Choi D: Solanaceae genomics: Current status of tomato (Solanum lycopersicum) genome sequencing and its application to pepper (Capsicum spp.) genome research. Plant Biotechnology 2007, 24:11-16.

21. Peters SA, Datema E, Szinay D, van Staveren MJ, Schijlen EG, van Haarst JC, Hesselink T, Abma-Henkens MH, Bai Y, de Jong H, Stiekema WJ, Klein Lankhorst RM, van Ham RC: Solanum lycopersicum cv. Heinz 1706 chromosome 6: distribution and abundance of genes and retrotransposable elements. Plant J 2009, Published Online, PMID: 19207213

22. Yoo EY, Kim S, Kim YH, Lee CJ, Kim BD: Construction of a deep coverage BAC library from Capsicum annuum, CM334. Theor Appl Genet 2003, 107:540-543.

23. Fulton TM, Van der Hoeven R, Eannetta NT, Tanksley SD: Identification, analysis, and utilization of conserved ortholog set markers for comparative genomics in higher plants. Plant Cell 2002, 14:1457-1467.

24. Jurka J, Kapitonov W, Pavlicek A, Klonowski P, Kohany O, Walichiewicz J: Repbase Update, a database of eukaryotic repetitive elements. Cytogenet Genome Res 2005, 110:462-467.

25. Jurka J, Klonowski $\mathrm{P}$, Dagman V, Pelton P: Censor-a program for identification and elimination of repetitive elements from DNA sequences. Comput Chem 1996, 20:119-121.

26. Xiong $Y$, Eickbush TH: Origin and evolution of retroelements based upon their reverse transcriptase sequences. EMBO J 1990, 9:3353-3362.

27. Lloréns C, Futami R, Bezemer D, Moya A: The Gypsy Database (GyDB) of mobile genetic elements. Nucleic Acids Res 2008, 36:D38-D46.

28. Jacobs SA, Khorasanizadeh S: Structure of HP1 chromodomain bound to a lysine 9-methylated histone H3 tail. Science 2002, 295:2080-2083.

29. Gao X, Hou1 Y, Ebina H, Levin HL, Voytas DF: Chromodomains direct integration of retrotransposons to heterochromatin. Genome Res 2008, 18:359-369.

30. Pereira $V$ : Insertion bias and purifying selection of retrotransposons in the Arabidopsis thaliana genome. Genome Biol 2004, 5:R79.

31. Friesen N, Brandes A, Heslop-Harrison JS: Diversity, origin, and distribution of retrotransposons (gypsy and copia) in conifers. Mol Biol Evol 2001, 18:1176-1188.

32. Natali $L$, Santini $S$, Giordani T, Minelli S, Maestrini P, Cionini PG, Cavallini A: Distribution of Ty3-gypsy- and Ty1-copia-like DNA sequences in the genus Helianthus and other Asteraceae. Genome 2006, 49:64-72.

33. SanMiguel $P$, Bennetzen $J$ : Evidence that a recent increase in maize genome size was caused by the massive amplification of intergene retrotransposons. Ann Bot 1998, 82:37-44.
34. Piegu B, Guyot R, Picault N, Roulin A, Saniyal A, Kim H, Collura K, Brar DS, Jackson S, Wing RA, Panaud O: Doubling genome size without polyploidization: Dynamics of retrotransposition-driven genomic expansions in Oryza australiensis, a wild relative of rice. Genome Res 2006, 16:1262-1269.

35. Vitte $C$, Bennetzen $\mathrm{J}$ : Analysis of retrotransposon structural diversity uncovers properties and propensities in angiosperm genome evolution. Proc Natl Acad Sci 2006, 103:17638-17643.

36. Zhang X, Wessler SR: Genome-wide comparative analysis of the transposable elements in the related species Arabidopsis thaliana and Brassica oleracea. Proc Natl Acad Sci 2004, 101(15):5589-5594.

37. Town CD, Cheung F, Maiti R, Crabtree J, Haas BJ, Wortman JR, Hine EE, Althoff R, Arbogast TS, Tallon LJ, Vigouroux M, Trick M, Bancroft I: Comparative genomics of Brassica oleracea and Arabidopsis thaliana reveal gene loss, fragmentation, and dispersal after polyploidy. Plant Cell 2006, 18(6):1348-1359.

38. Ewing B, Green P: Base-calling of automated sequencer traces using phred. II. Error probabilities. Genome Res 1998, 8:186-194.

39. Salamov AA, Solovyev W: Ab initio gene finding in Drosophila genomic DNA. Genome Res 2000, 10:516-522.

40. Nix DA, Eisen MB: GATA: a graphic alignment tool for comparative sequence analysis. BMC Bioinformatics 2005, 6, Published online (PMID: 15655071).

41. Eddy SR: Profile hidden Markov models. Bioinformatics 1998, 14:755-763.

42. Xu Z, Wang H: LTR_FINDER: an efficient tool for the prediction of fulllength LTR retrotransposons. Nucleic Acids Res 2007, 35:265-268.

43. Park J, Park B, Jung K, Jang S, Yu K, Choi J, Kong S, Park J, Kim S, Kim H Kim S, Kim JF, Blair JE, Lee K, Kang S, Lee YH: CFGP: a web-based, comparative fungal genomics platform. Nucleic Acids Res 2008, 36 D562-D571.

44. Tamura K, Dudley J, Nei M, Kumar S: MEGA4: Molecular Evolutionary Genetics Analysis (MEGA) software version 4.0. Mol Biol Evol 2007, 24:1596-1599.

45. Koo DH, Plaha P, Lim YP, Hur Y, Bang JW: A high-resolution karyotype of Brassica rapa ssp. pekinensis revealed by pachytene analysis and multicolor fluorescence in situ hybridization. Theor Appl Genet 2004 109:1346-1352

46. Kwon JK, Kim BD: Localization of $5 \mathrm{~S}$ and $25 \mathrm{~S}$ rRNA genes on somatic and meiotic chromosomes in Capsicum species of chili pepper. Mol Cells 2009, 27:205-209.

doi:10.1186/1471-2164-12-85

Cite this article as: Park et al:: Comparative analysis of pepper and tomato reveals euchromatin expansion of pepper genome caused by differential accumulation of Ty3/Gypsy-like elements. BMC Genomics 2011 $12 \cdot 85$

\section{Submit your next manuscript to BioMed Central and take full advantage of:}

- Convenient online submission

- Thorough peer review

- No space constraints or color figure charges

- Immediate publication on acceptance

- Inclusion in PubMed, CAS, Scopus and Google Scholar

- Research which is freely available for redistribution 